\title{
Poetologie im Lehrplan des Germanistik-Studiums
}

\section{Zwei Sichtweisen}

\author{
Doris Flohr (D), Stadthagen - Otto Holzapfel (D), Freiburg i. Br.
}

https://dx.doi.org/10.37583/diyalog.759432

\begin{abstract}
Deutsch)
Eine Lehrveranstaltung ,Poetologie“ sollte zum Lehrplan ,Deutsch als Fremdsprache“ in der LehrerInnenausbildung in der Türkei gehören. Doch wie schaffen wir es, die Studierenden für Poetik, für Lyrik und dichterische Prosa zu begeistern, ohne sie mit trockenen Regelwerken und mit der Pflichtlektüre von Datenmengen abzuschrecken? Aus zwei verschiedenen Perspektiven versuchen wir das Problem zu beleuchten, das heißt den eigenen Standpunkt kritisch zu hinterfragen und Erfahrungen zu bewerten: erstens die Sicht einer Deutsch lehrenden deutschen Sprachvermittlerin für (unter anderen) türkische Schülerinnen und Schüler in Deutschland und, zweitens, die Sicht eines Deutsch lehrenden deutschen Gastprofessors in der Türkei. Wesentlich fehlt hier die ,dritte' (und eigentlich von der Gewichtung her die erste) Sichtweise, nämlich die der Deutsch lehrenden türkischen Person in der Türkei. Wir verzichten nicht bewusst darauf, aber wir möchten mit diesen Anmerkungen zum Nachdenken anregen. ${ }^{1}$
\end{abstract}

Schlüsselwörter: Poetologie (Lehre von Poetik, Lyrik und dichterischer Prosa); Interesse wecken, subjektive Erfahrungen und Perspektiven dazu

\section{Abstract (English) \\ Poetology in the curriculum of German studies: two perspectives}

A course 'Poetology' could be part of the curriculum in the educational training of teachers of German language and literature in Turkey. But how do we motivate students about poetics, poetry and poetic prose without deterring them with dry rules and with the mandatory reading of data? We try to illuminate the problem from two different perspectives, i.e. to examine critically our own point of view and to evaluate experiences: firstly, the view of a German language mediator teaching German for (among other things) Turkish students in Germany and, secondly, the view of a German guestprofessor teaching German in Turkey. The missing point here is the perspective of a 'third' person, namely that of the Turkish person teaching German in Turkey, which should have been the first priority. We do not consciously forego this, but we would like to encourage one to think about the obvious problems with these comments.

Keywords: poetology (teaching poetics, poetry and poetic prose); individual experiences

${ }^{1}$ Dazu gibt es sicherlich bereits Untersuchungen; mir [Otto Holzapfel] ist bekannt [allerdings nicht gelesen] Gencer Çıtak, Özlem (2019): Zur Entwicklung von Gesprächskompetenzen im Deutschen als Fremdsprache. Eine longitudinale Studie anhand der Germanistikstudenten in der Türkei. Berlin: Logos Verlag (Germanistik in der Türkei, 5). ISBN 978-3-8325-5035-6. 


\section{EXTENDED ABSTRACT}

A poetics course should be part of the curriculum for "German as a foreign language" in Turkey. It would be important to awaken the students' enthusiasm for poetry and poetic prose without putting them off with dry rules. - We write about this problem from two perspectives and try to critically consider our own points of view. First, it is the view of a German teaching language mediator for students, including students of Turkish origin in Germany, secondly, the view of a German guest professor teaching German in Turkey. The third point of view is essentially missing here, namely that of the Turkish person teaching German in Turkey. We do not consciously forego this, but we would like to encourage the discussion of our comments. We believe that this is necessary and we hope that it will be fruitful - especially at a time of virtual teaching, when digital access to learning materials is becoming more necessary than ever. The current situation offers an opportunity to think about what seems really important when studying German literature.

The background for Doris Flohr's first perspective is the experience of teaching German at a high school in France. There were difficulties in understanding the texts, so that free and personal engagement with poetry was neglected. The language itself was the problem; literature played practically no role. It was not much different in Germany with French courses for Germans. However, as a German teacher in Moscow for a short time, Ms. Flohr experienced that with excellent language skills, the interest in classic German literature was also considerable. - In language classes for refugee children at a high school in Germany, different and new learning goals have to be worked out. It is of little use to set regulations, when conditions differ. In addition to language teaching, regional history can also play an important role. But poetry is suitable for being emotionally moved by a text, and we consider this an important task in the sense of the Germanist Emil Staiger: "To understand what moves us." - Lessons should consistently take place in the foreign language. Not using your own language is a challenge for both students and teachers. - The joy of students in literary texts can also be awakened with folk songs, modern songs and contemporary poems.

The explanations on the second perspective draw on experience gained from teaching German in Turkey as a German professor at the university and realizing that teaching experience at a German university does not always help in that case. Otto Holzapfel had to learn that this cannot necessarily be explained by a qualitative difference. Students in Germany have different reading experiences, and other tools are available to them. In digital teaching, such differences can be compensated over time, and in the current situation, this can be seen as an opportunity. The world wide web will continue to play an essential role in the future, and it will be an urgent task to compensate for lack of direct contact through intensive personal support. The role of the lecturer could change, as tutoring and stimulation become more important than learning materials. These could also be prepared and communicated centrally. The fact that the teacher can arouse and promote enthusiasm may play a key role.

According to Holzapfel's experience, Turkey offers excellent conditions for poetology. Here, the teacher encounters joy and immediacy, accepting poetic feeling and thinking. This is an opportunity that can be used. Although the author cannot contribute any theory to this, experiences with simple poems, for example with C. F. Meyer's "The Roman Fountain" were promising. The same applies to a spontaneously compiled collection of autumn poems. The students could go out to feel the autumn wind for example as homework, in order to 
"understand" the rain, the storm and the joy of harvesting, which speaks from the corresponding poems. Poems about nature are based on such experiences, and can also convey them wonderfully as texts, sharpening the aesthetic awareness of literary work in general, and that seems important. Even everyday life in Germany, idealized and transfigured for example in the bourgeois poem by J. G. von Salis-Seewis with his "Colorful are the forests..." can be brought to life and experienced. - Love poetry need not be left out. The fact that there were Arabic models in Andalusia for French and German minnesong could awaken appreciation for this influence, which played a major role in European culture. - The teacher must rather point out paths to get there. Learners must try to acquire the knowledge of a language and literature with an enthusiasm for discovery. Poetology is definitely one way of getting closer to literature and language. 


\title{
Einleitung
}

Der folgende Beitrag ist ein Erfahrungsbericht, der ursprünglich als Dialog mit der ,dritten“ Perspektive gedacht war, nämlich zur Anregung eines Freundes und Kollegen in der Türkei, eine vorzuschlagende Lehrveranstaltung ,Poetologie ${ }^{`} \mathrm{zu}$ begründen und aufzuwerten. Leider ist dieser dritte, notwendigste Teil nicht zustande gekommen. Trotzdem möchten wir hiermit eine unseres Erachtens dringende Diskussion anregen, gerade auch in einer Zeit [März 2020], in der wir in Deutschland durch die Corona-Krise alle sozialen Kontakte auf ein Minimum eingeschränkt haben. Schulen und Universitäten sind geschlossen, und man denkt z. B. darüber nach, die laufenden Abiturvorbereitungen ausfallen zu lassen und die bisherige Notengebung ( $2 / 3$ werden auf die Endnote angerechnet) ohne weitere mündliche und schriftliche Prüfung gelten zu lassen. Vielleicht müssen wir uns mittel- oder sogar langfristig auf alternative Lehr- und Prüfungsformen einstellen. In dieser Hinsicht lohnt es sich darüber nachzudenken, was uns am Studium der deutschen Sprache und Literatur wichtig erscheint. Insofern ist dieser Beitrag auch keine wissenschaftlich fundierte Studie, sondern eine Anregung zur Diskussion. Manchen der folgenden Aussagen werden LeserInnnen nicht unmittelbar zustimmen wollen; vieles davon ist individuell bedingt und subjektiv. Leidet darunter die ,Ehrlichkeit‘ der Argumente? Statistikgestützte Fallstudien können wir nicht vorlegen, aber hoffentlich zur Diskussion über die angeschnittenen Fragestellungen anregen.

\author{
Perspektive ,eins ${ }^{6}$ \\ Ansteckungsgefahr \\ Wie wunderbar, im Buch zu lesen, \\ was einst in Dichters Kopf gewesen \\ und nun in meinen rüber will. \\ Ich bin gespannt und halte still. \\ $D a$, jetzt, jetzt ist es angekommen \\ oder war es schon vorher da?
}

Dem Dichter bleibt es unbenommen:

Kunst steckt uns an wie Cholera.

Jochen Jung (geboren 1942)

Von 1963 bis 1968 habe ich [Doris Flohr] Germanistik und Romanistik und ein bisschen Slawistik studiert. In meinem Studium habe ich mich gewundert, dass die Textbesprechungen in Romanistik auf Deutsch stattfanden, während wir in der Schule unsere Lektüre auf Französisch erklärt hatten. - Von 1966 bis 1967 war ich ,assistante' an einem Gymnasium in Blois/Frankreich. Ich habe dort den Deutschunterricht unterstützt und an einer Art Volkshochschule Deutsch für Erwachsene unterrichtet. Das Textverständnis beanspruchte den größten Teil des Schulunterrichts. Eine freie und eigene Auseinandersetzung mit den Texten, wie ich es aus meinem eigenen Schulunterricht kannte, war nicht vorgesehen: Die SchülerInnen sollten zuerst die Sprache, Texte und Fakten lernen. Der Deutschunterricht für Erwachsene hatte dagegen mehr praktische Ziele: Die TeilnehmerInnen wollten sich in 
Deutschland zurechtfinden und sich mit Deutschen unterhalten können. Die Literatur spielte da praktisch keine Rolle. Dasselbe habe ich in späteren Jahren in Deutschland bei meinen Französischkursen für Deutsche erlebt. Hin und wieder gab es Ausnahmen: Einzelne TeilnehmerInnen wollten z.B. die Texte französischer Chansons kennen lernen.

In den Jahren von 1990 bis 1994 habe ich Spätaussiedler und jüdische Kontingentflüchtlinge aus der ehemaligen Sowjetunion unterrichtet. Da hatte ich es mit ganz heterogenen Gruppen zu tun: vom Analphabeten bis zum Akademiker. Wir haben versucht, den Unterricht entsprechend zu differenzieren. Gedichte habe ich manchmal auf Wunsch neben dem Unterricht verteilt oder mit interessierten TeilnehmerInnen über Kunst, Musik und Literatur gesprochen. Sie brachten auch russische Lieder und Chansons mit, die wir dann auf Deutsch übersetzten. - 1992 und 1993 war ich als Deutschlehrerin je einen Monat in Moskau: einmal für Deutschlehrende aus der ganzen ehemaligen Sowjetunion, das zweite Mal für Menschen, die mit Deutschland Handel treiben wollten. Die Deutschlehrenden verblüfften mich durch ihr hervorragendes Deutsch, selbst wenn sie noch nie in Deutschland gewesen waren, und durch ihre Kenntnis der klassischen deutschen Literatur. Sie suchten den lebendigen Anschluss an die Gegenwartssprache und an die zeitgenössische Literatur. Beliebt waren auch Liedermacher wie z.B. Udo Lindenberg. Die Kaufleute verfolgten ganz konkrete Ziele: Sie suchten das Vokabular des Alltags, der Politik und der Wirtschaft und hätten mich sogar gerne als Repräsentantin ihrer Geschäftszweige in Deutschland gesehen.

Von Januar 2016 bis Sommer 2018 habe ich in den Sprachlernklassen für syrische und irakische Flüchtlingskinder in einem Gymnasium mitgearbeitet. Die Kinder hatten viel Freude an Kindergedichten mit Lautmalerei und Reimen und konnten sie schnell auswendig. Seit 2016 begleite ich eine yesidische Schülerin aus dem kurdischen Teil des Irak. Sie möchte hier Abitur machen und nimmt am normalen Unterricht der Oberstufe teil. Es fällt ihr schwer, sich in allen möglichen Fächern zu entwickeln und zu den verschiedensten Themen ihre eigene Meinung zu äußern. Im Irak wurden Gedichte vor allem auswendig gelernt, vielleicht wurde auf besondere Merkmale und Schönheiten hingewiesen, aber die ,Gedichtinterpretation ' mit vielen rhetorischen Figuren ist für sie eine große Herausforderung. Der erste Schritt ist für sie immer noch das Textverständnis: Wie soll ich zum Beispiel Neologismen (künstliche Sprachbildungen) erkennen, sagt sie beispielsweise, wenn der Text für mich voller ,Neologismen'[unbekannte Wörter] ist?

Ich habe das alles erzählt, um zu zeigen, wie unterschiedlich meine Erfahrungen mit dem Sprachunterricht sind. Es war mir immer hilfreich, wenn ich die Sprache der Teilnehmer kannte, Französisch, zum Teil Englisch, ein bisschen Russisch. Dann sind typische Fehler beim Weg von der einen Sprache in die andere leichter zu erkennen: „Ich arbeite wie Pferd“, sagt vielleicht jemand aus Russland, wo es keinen Artikel gibt. Bei den Flüchtlingskindern fehlte diese gemeinsame Sprache. Lehrer mit Arabisch- oder Kurdischkenntnissen sind selten - und das Internet hilft bei Vokabeln. Auf die Dauer merkt man jedoch die typischen Strukturfehler, zum Beispiel in der Deklination oder im Satzbau.

Ich habe auch türkische Schüler begleitet, privat als Hausaufgabenhilfe. Es waren vor allem zwei sehr begabte junge Frauen, die ganze Redewendungen und Ausdrucksweisen schnell gelernt und angewandt haben. Beide haben ein Hochschulstudium (Betriebswirtschaft bzw. Pädagogik) mit sehr guten Abschlüssen beendet, sie sind beruflich erfolgreich und praktisch zweisprachig. - Für ein Germanistikstudium in der Türkei geht es wahrscheinlich 
um andere Aufgaben, als ich es erlebt habe, vergleichbar mit meinen eigenen Französischund Russischstudien: Sprachvermittlung, Landeskunde (Geographie, Geschichte, Politik ...), Literatur und Kunst, also auch Musik und bildende Kunst. Gibt es dafür die notwendigen Hilfsmittel, oder muss der Dozent das Material selbst zusammenstellen? Gibt es einen verbindlichen Kanon, oder ist der Dozent frei in der Auswahl der Texte? Wenn man zu eng an Vorschriften gebunden ist, muss man andere Wege der zusätzlichen Vermittlung literarischer Werte suchen. Wahrscheinlich bietet der obenstehende Vorschlag zur ,Poetologie eine gute Chance in dieser Richtung. Lyrik eignet sich dazu, von einem Text ,ergriffen' zu werden, emotional und einfach, aus Interesse' an Form und Inhalt. Das zu vermitteln, müsste eine Aufgabe für die Lehrenden sein. Der Germanist Emil Staiger (1908 - 1987) hat einmal als Ziel der Interpretation formuliert: ,Begreifen, was uns ergreift." Man kann das in unterschiedlicher Weise verstehen, aber vor allem muss man zulassen, sich von einem Text ,ergreifen` zu lassen.

Diese berühmte ,Formel' wird vielfach in Verbindung mit Emil Staiger zitiert, z. B. von seinem Schüler Adolf Muschg im Nachruf auf Staiger in „Die Zeit“" vom 8. Mai 1987, Nr. 20 (www.zeit.de/1987/20/begreifen [...]; aufgerufen im Juni 2020) und ebenso in dem Artikel über Staiger in der deutschen Wikipedia (de.Wikipedia.org; aufgerufen im Juni 2020). Eine direkte Zitatstelle bei Staiger wurde nicht verifiziert.

Ich fände es auch wünschenswert, dass (soweit möglich) die Textinterpretation auf Deutsch stattfindet. Mit Hilfe vorgegebener Begriffe ist das durchaus möglich. Es scheint mir wichtig, in möglichst allen Bereichen des Unterrichts Sprechfertigkeit in der zu erlernenden Fremdsprache zu vermitteln und konsequent auf die Verwendung der eigenen Sprache zu verzichten. Das ist sowohl für die Studierenden wie auch für die Lehrenden eine Herausforderung, der sie sich aber stellen sollten.

Gedichte eignen sich wegen ihrer Kürze gut für einen Überblick über die Geschichte der deutschen Literatur, aber natürlich sollten die Studierenden auch Volkslieder, moderne Lieder und zeitgenössische Gedichte kennen lernen. Für mich wäre es das erste Ziel, Freude an den Gedichten zu wecken; ein nächster Schritt wäre es, die künstlerischen Mittel zu erkennen und benennen zu können. Und sehr wünschenswert finde ich es, Gedichte und Lieder auswendig zu lernen. Das ist ein Schatz, den man immer bei sich trägt. Früher war das in Deutschland üblicher als heute. Ältere Menschen, die oft nur die Volksschule besucht haben, besitzen einen Reichtum an solchen Texten, den ich immer wieder bewundere. - Dafür dürfen die Gedichte nicht zu lang und nicht zu schwer sein. Man sollte einen Fundus an Liedern und Gedichten haben, nach Schwierigkeit und Themen geordnet, aus dem man je nach Können und Interesse der Studierenden auswählen kann. Beispiele:

Lustige, spielerische Texte von Wilhelm Busch, Heinrich Heine, Christian Morgenstern, Ernst Jandl usw.; Liebeslyrik vom Mittelalter (Du bist mîn...) über Barock und die weiteren Literaturepochen bis heute; Naturlyrik; Themen wie Großstadt, Krieg, Tod...; Nationalhymnen im Vergleich; Volks-und Kinderlieder, zum Beispiel Bunt sind schon die Wälder... Grün, grün, grün sind alle meine Kleider... Von diesem Lied gibt es zahlreiche Varianten; durch die Wiederholungen ist es leicht zu singen und erfreut mit dem Durchspielen der Farben. Man kann es beliebig erweitern und lernt dabei spielend verschiedene Berufe kennen. 
1.Grün, grün, grün sind alle meine Kleider,

grün, grün, grün ist alles, was ich hab'.

Darum lieb' ich alles, was so grün ist,

weil mein Schatz ein Förster ist.

2.Weiß, weiß weiß sind alle meine Kleider,

... weil mein Schatz ein Bäckermeister ist.

3.Rot, rot, rot sind alle meine Kleider,

... weil mein Schatz ein Feuerwehrmann ist.

4.Blau, blau, blau sind alle meine Kleider,

... weil mein Schatz ein Matrose ist.

5.Schwarz, schwarz, schwarz sind alle meine Kleider,

... weil mein Schatz ein Schornsteinfeger ist.

\section{Perspektive ,zwei ${ }^{6}$}

Die Erfahrungen, die eine in der Türkei lehrende deutsche Person macht, werden andere sein als jene, die einer türkischen Lehrkraft dort begegnen. Zumindest sind die jeweiligen Blickwinkel sehr unterschiedlich, wobei ich [Otto Holzapfel] als Deutscher darauf achten muss, dass ich die Studierenden in der Türkei nicht falsch einschätze, zumal, wenn ich Lehrerfahrungen an einer deutschen Universität habe und diese vorschnell auf meine Erwartungen für die Türkei übertrage. Das muss man nicht unbedingt mit einem qualitativen Unterschied gleichsetzen, aber zweifellos haben Studierende in Deutschland andere Vorbedingungen für ihre Ausbildung als jene in der Türkei. Zumindest die Generation, die (wie ich) noch ohne Smartphone und Internet aufgewachsen ist, hat eine andere Basis in der Leseerfahrung, und auch in der Gegenwart steht jedem Universitätsangehörigen in der Regel sowohl eine umfangreiche Seminarbibliothek wie auch eine Uni-Bibliothek zur Verfügung. Und selbst wenn Buchläden heute zunehmend Probleme bekommen, ist das Angebot an Editionen zu einem günstigen Preis, an billigen Nachdrucken und an antiquarischen Exemplaren auch in der Fachliteratur weiterhin überwältigend. Wenn es nur um literarische Werke geht und um Handbücher zur Literaturwissenschaft, dann bietet bereits jede städtische Ausleihbibliothek eine reiche Auswahl. Wir sind nicht unbedingt allein auf Wikipedia.org angewiesen, finden aber hier leicht einen ersten Zugang, der ausgebaut und weiterverfolgt werden kann. Und es gibt zahllose spezielle Internetseiten, die man konsultieren kann.

Das gilt unabhängig von den wechselnden Erfahrungen, die man mit Wikipedia machen kann und die einen oftmals sehr skeptisch werden lassen. Gerade dieses Medium ist (wie grundsätzlich alle Internet-Seiten) kritisch und im Vergleich mit anderen Quellen zu prüfen. ${ }^{2}$ Aber gleiches gilt für manche Edition oder sogar für ein Fachbuch, das sich (nach Ablauf des Urheberrechts) im Nachdruck zuweilen als ,neu' tarnt und Erwartungen bezüglich des aktuellen Standes der Wissenschaft nicht erfüllen kann. Andererseits ist die Situation in den Geisteswissenschaften anders als in den Naturwissenschaften, wo Fachbücher oft nach einer kurzen Zeitspanne überholt erscheinen, während dem Philologen und Germanisten ein

\footnotetext{
${ }^{2}$ Holzapfel, Otto (2016): „Erfahrungen mit Wikipedia.de“. In: [türkische Internet-Zeitschrift] Diyalog. Interkulturelle Zeitschrift für Germanistik 2016/1, S. 99-118.
} 
älteres Fachbuch oft wichtige Einsichten vermitteln kann. ${ }^{3}$ Das alles bedeutet, dass wir meines Erachtens in Deutschland in einem geistigen Milieu leben, das (trotz Internet usw.) weithin mit ,Buchwissen' gefüllt ist- manchmal anscheinend sogar überfüllt ist, wenn man an den gegenwärtigen Umfang von Dissertationen und Habilitationsschriften denkt. Das sind oft Tausende von Seiten, während meine eigene Dissertation 1969 mit mageren 157 Seiten auskam. Hoffen wir, dass das auch eine qualitative Erweiterung bedeutet; manchmal scheint es nicht so. Umfang ist nicht mit Qualität gleichzusetzen. ${ }^{4}$

In einem Interview, das ich im Januar 2019 zufällig mithörte, berichtet eine französische Professorin, die in Berlin lehrt, von ihren Erfahrungen und meint u.a., dass sie die heutigen (deutschen) Studierenden als sehr kritisch erlebe. ,Sie wissen nicht viel, aber sie hinterfragen kritisch, was ich ihnen vorsetze. ${ }^{5}$ Das ist einerseits kein Lob für deutsche Studierende, die ,Wissen` zur Verfügung haben, es aber vielleicht nicht genügend benutzen. Andererseits kann man dafür argumentieren, dass eine kritische Haltung Voraussetzung dafür ist, mit dem vorhandenen, Wissen" (aus Büchern und Internet) richtig umzugehen. Voraussetzung dafür ist, dass ,Wissen“ auf allen Ebenen und mit allen Medien frei zur Verfügung steht.

Wir haben in Deutschland ebenfalls eine gewisse Tradition dafür, dass wir ein Buch in die Hand nehmen können und es mit den erworbenen Erfahrungen über den Autor, den Verlag, die Ausstattung (Register, Literaturverzeichnis, Quellenangaben), die Zitierweise usw. kritisch beurteilen. Das droht (und drohte tatsächlich konkret an vielen Stellen ${ }^{6}$ ) dort verloren zu gehen, wo man, wie das einmal als sehr praktisch galt, die Studierenden nur mit Kopien versorgte, welche dann ,Pflichtlektüre' darstellten. Ein solches Vorgehen fokussiert ausschließlich auf den ungeprüften Mitteilungswert eines Textes und übersieht, dass es zur Wissenschaft gehört, mit jedem fachlichen Text kritisch umzugehen und sich selbst im Vergleich mit anderen Quellen eine Meinung zu bilden. Das kann nur im Kontext mit mehreren Kriterien geschehen.

Das alles habe ich in der Türkei ,ziemlich anders` erlebt. Aber es wäre verfrüht, dem hier nur eine negative Bilanz gegenüber zu stellen. Dem mit Buchwissen ,überfütterten“ Studierenden bei uns möchte ich eine Personentypik entgegenstellen, die man in der Haltung als ,naiv' bezeichnen kann, im positiven Sinne auch als ,unvoreingenommen'. Was hier an Erfahrung und an Möglichkeiten, Erfahrungen zu machen, fehlt, erweist sich aus einem anderen Blickwinkel nämlich durchaus als Vorzug. Wann und wo hätte ich erlebt, dass

\footnotetext{
${ }^{3}$ Holzapfel, Otto (2018): „Vom Vergnügen ein älteres Fach zu lesen. Eine Diskussion im Grenzgebiet von Natur- und Geisteswissenschaft“. In: [türkische Internet-Zeitschrift] Anadolu University Journal of Education Faculty (AUJEF), Special Issue August 2018 = Festschrift Seyyare Duman, S. 179-184.

${ }^{4}$ Und - leider aus der Erfahrung in der Türkei: Eine Examensarbeit, die fleißig Kopien sammelt und abheftet, hat kaum wissenschaftlichen Wert. Diese Aussage missfällt in einem Gutachten zu diesem Beitrag. Das ist verständlich, aber auch das ist leider Erfahrung: Kopiertätigkeit bei Hausarbeiten und bei Examensarbeiten, die durch Umfang die eigene Leistung, zudecken“.

${ }^{5}$ Ich kann sie nicht wörtlich zitieren; daher spielen Namen und Quelle hier keine Rolle.

${ }^{6}$ Das habe ich z. B. an den Universitäten von Kopenhagen (Dänemark) und Lund (Schweden) in den 1970er und 1980er Jahren erlebt. In Schweden wurden die Bedingungen für das Studium drastisch verringert, so dass ,jede“ und ,jeder' studieren konnte. Aber da musste man sich dann mit Kopien behelfen, weil das ganze Buch ,zu anstrengend' und angeblich ,unnötig' gewesen wäre, und - noch überraschender - dänische Texte wurde in das Schwedische übersetzt (die beiden Sprachen liegen sich ähnlich nahe wie unterschiedliche deutsche Dialekte). An Fremdsprachen (Englisch, Französisch) war da offensichtlich überhaupt nicht zu denken.
} 
Studierende in Deutschland sich bei mir mit einem selbstgeschriebenen Gedicht bedanken, sich trauen, auf den Lehrenden ein eigenes Gedicht zu verfassen? Hier trat mir in der Türkei eine ungeahnte Freude und Unmittelbarkeit entgegen, poetisches Fühlen und Denken zu akzeptieren und sogar selbst auszuprobieren. In Deutschland scheint mir dagegen alles eher ,bereits gesagt ${ }^{\star}$ und, längst überholt', bereits wieder verworfen und auf der Ebene der akademischen Lehre und Forschung, aber auch im öffentlichen Leben vor allem vom Zwang eingeengt und bedrängt, immer, etwas Neues' ${ }^{6} \mathrm{zu}$ formulieren und präsentieren zu müssen. Das ist für mich eine Entwicklung, die besonders in der bildenden Kunst deutlich wird. Manche Ergebnisse können einem Unvoreingenommenen ehrlicherweise nur ein Kopfschütteln abverlangen (aber das behält man zumeist lieber für sich). Nicht parallel dazu, aber mit vergleichbaren Auswirkungen, ist der Trend (in der Gegenwart vorherrschend, aber von mir so erlebt in Dänemark in den 1970er Jahren), dass Wissenschaft zur ,Projektforschung' wird, dass nur dafür Geld bewilligt wird, was ,überraschend und neu' ist und dass jener an der Universität am meisten gilt, der die höchste Summe an Fremdmitteln einsammelt. Kontinuität ist nicht gefragt; kaum ist ein Projekt zu Ende, arbeitet man bereits für das nächste, und die laufende akademische Lehre leidet offensichtlich darunter. ,Projekte“ sind in der Regel nur für eine kleine Schar von Forschern nützlich (ja sogar sehr nützlich), aber der breiten Masse der Studierenden kommt das kaum zu Gute. Also wäre meine Hoffnung, dass dieser Weg an türkischen Universitäten nicht beschritten wird (dafür kann es spezielle Forschungseinrichtungen geben, die man gesondert finanzieren sollte), sondern dass der Schwerpunkt die Bildung und Ausbildung der Mehrzahl der Studierenden bleibt. Kehren wir nach diesen generellen Voraussetzungen zum eingeengten Problem des Germanistik-Studiums und der Ausbildung von Deutschlehrenden in der Türkei zurück. ${ }^{7}$ Und ich versuche auf den Bereich der ,Poetologie‘ zu fokussieren.

Hier wäre es mein Wunsch, aus der Perspektive der türkischen Studierenden die ursprüngliche Unvoreingenommenheit, von der ich oben sprach, zu erhalten, zu bewahren, und sie mit neuen Erfahrungen zu ergänzen, aber auf keinen Fall zu verdrängen. Ich bewege mich dabei zugegebenermaßen auf einem problematischen Feld, und ich kann dazu kaum Erfahrungen, sondern nur Theoretisches und das nur versuchsweise beitragen. Meine Argumentation kann ich aber mit konkreten Beispielen zu verdeutlichen versuchen. Türkische Studierende in den Anfangssemestern mit Gedichten zu konfrontieren, die ein hohes Interpretationsniveau erfordern, halte ich für verfehlt. Die Texte, mit denen erste Erfahrungen zu machen sind, dürfen vom Inhalt her eher einsichtig sein und ebenso einfache Strukturen aufweisen. Das ist in der Regel bei dem sogenannten Dinggedicht der Fall. Als Beispiel wählte ich in Çanakkale das Gedicht ,Der römische Brunnen' von Conrad Ferdinand Meyer (1825-1898) aus dem Jahre 1882, in dem er die ,Fontana dei Cavalli Marini‘ in der Villa Borghese in Rom beschreibt:

\footnotetext{
${ }^{7}$ Ich versuche aus Erfahrungen zu formulieren in Konya im Sommer-Semester 1991: eine kurze DAADGastdozentur: „Europäische Volksballadenforschung“ und „Das deutsche Volkslied als literarische Gattung“: Vorlesungen und begleitende Seminare (in Wirklichkeit reichte es nur für einen improvisierten Deutschunterricht) und Februar bis Juni 2006, nach meiner Pensionierung, ein Aufenthalt an der türkischen Universität von Çanakkale mit Lehrveranstaltungen in Vertretung meines Kollegen Ali Osman Öztürk: „Sprechfertigkeit Deutsch“ (2. Semester), „Analyse und Didaktik deutscher Lyrik“ (8. Semester) und „Magisterkolloquium: Kunstballaden und Volksballaden“. In der Realität war der Schwerpunkt improvisierter Deutschunterricht in (fast) allen vier Klassen, der mir aber große Freude bereitete.
} 


\author{
Auf steigt der Strahl und fallend gießt \\ er voll der Marmorschale Rund, \\ die, sich verschleiernd, überfließt \\ in einer zweiten Schale Grund; \\ die zweite gibt, sie wird zu reich, \\ der dritten wallend ihre Flut, \\ und jede nimmt und gibt zugleich \\ und strömt und ruht.
}

Formal kann man auf den einfachen Wechsel der endreimenden Zeilen verweisen, und an diesem Beispiel muss man nicht das komplizierte Geflecht der deutschen Grammatik mit einer Flut von Fremdwörtern und Begriffen analysieren, unter denen sich die Studierenden zumeist wenig vorstellen können - vermutlich manche Lehrende ebenso. Das hat mich eher abgestoßen: diese Definitionssucht und dieses Bemühen um strenge Kategorisierungen, die ich im türkischen Germanistik-Unterricht erlebt habe, und ich halte das für einen verfehlten Weg, mit Dichtung umzugehen. Gut: Regeln müssen sein - und man muss diese auch verstehen und erlernen. Aber Sprache und Literatur sind, poetologisch gesehen, nicht Texte, die einem Regelwerk folgen, sondern Phänomene eines elementaren Erlebnisses und einer Vermittlungsform, an der ästhetische Qualitäten entdeckt werden sollten und die ,Welterfahrung', die ein solches Gedicht mitteilt - die Erfahrung, wie sie eben ein Dichter macht und ausdrückt.

Überraschend für mich war, wie es den Studierenden offensichtlich schwer fiel, sich das Bild vorzustellen, welches das Gedicht beschreibt. Ich bat sie an der Tafel einen solchen Brunnen zu zeichnen und immer wieder gab es nur Versuche, einen der üblichen türkischen Röhrenbrunnen darzustellen: Aus einer Wand bieten eine oder mehrere Röhren den direkten Wasserstrahl. Mein eigener Versuch, den dreischaligen Brunnen zu zeichnen, war nicht überzeugend, und erst in der nächsten Stunde konnte ich eine korrekte Abbildung vorzeigen (zwar nicht den Borghese-Brunnen, aber eine ähnliche Konstruktion mehrerer Schalen übereinander). Darauf war ich nicht vorbereitet, und es lehrte mich, dass ich die Studierenden selbst in solchen ,einfachen“ Dingen dort ,abholen' muss, wo sie ihre eigenen Erfahrungen einbringen können. - Ein nächster Schritt war es, dem Weg des fließenden Wassers mit Armbewegungen nachzuspüren. Das Gedicht wollte nicht erklärt, sondern erlebt werden.

Ein weiterer Schritt, der mangels Zeit und mangels Unterlagen dazu leider entfallen musste, wäre gewesen, die verschiedenen Vorstufen C. F. Meyers zu dieser Endfassung miteinander zu vergleichen. ${ }^{8}$ Damit kann man verdeutlichen, was Dichtung bedeutet, in diesem Fall nämlich ganz konkret Verdichtung und Vereinfachung von Sprache und Form auf wenige Zeilen, die meiner Ansicht nach unübertroffen eine höchst engmaschige Textur präsentieren - und das mit den formal einfachsten Mitteln. - Gedichte als ,Erlebnisse' zu

\footnotetext{
${ }^{8}$ In Wikipedia.de ,Der römische Brunnen' [aufgerufen im Dezember 2018] sind zwei frühere Fassungen, die vierte von 1866 und die sechste von 1870 neben der endgültigen siebten von 1882 abgedruckt. Jene von 1866 ist auffallend länger, von deutlich geringerer ,Dichte` der Aussage und mit erklärungsbedürftigen Wörtern (Bronne, Geleucht, Laubesnacht): In einem römischen Garten / verborgen ist ein Bronne, / behütet von dem harten / Geleucht' der Mittagssonne, / er steigt in schlankem Strahle / in dunkle Laubesnacht / und sinkt in eine Schale / und übergießt sie sacht. / Die Wasser steigen nieder / in zweiter Schale Mitte, / und voll ist diese wieder, / sie fluten in die dritte: / Ein Nehmen und ein Geben, / und alle bleiben reich, / und alle Fluten leben / und ruhen doch zugleich.
} 
vermitteln, die nicht nur das Ohr und das Gehirn erreichen, sondern sozusagen das Herz: Das wäre ein Weg, den zu beschreiten sich meines Erachtens lohnt, und das durchaus als Gegengewicht zum sonstigen Literaturunterricht (der deswegen nicht herabgewürdigt sein soll). Da Lehrmaterial fehlte, versuchte ich eine Reihe von Gedichten zusammen zu stellen, die für eine Jahreszeit typisch erscheinen. Ich wählte (allerdings im Frühjahrssemester) Herbstgedichte. $^{9}$

Auffallend ist in der deutschen Tradition die Konzentrierung auf den Herbst gegenüber Gedichten, welche die anderen Jahreszeiten beschreiben. Von den Herbstgedichten gibt es nämlich in der deutschen Literatur eine reiche Anzahl ganz unterschiedlicher Art, die alle den Vorzug haben, dass ich das individuelle Gedicht mit dem vergleichen kann, was ich selbst in einem (,deutschen') Herbst erlebe. Ist es beim Dinggedicht der Gegenstand, so ist es hier etwa ein Gefühl, eine Stimmung. An die Studierenden: „Bitte gehen Sie als Hausaufgabe hinaus und spüren Sie den Herbstwind, den Regen, den Sturm, ,begreifen' Sie (mit den Händen) die Fülle der Obstsorten, die Freude an der Ernte - und versuchen Sie das in eigene Worte zu fassen (als Prosa oder Gedicht).“ Für türkische Verhältnisse müsste ähnlich Paralleles zu finden sein, etwa den abrupten Übergang vom fast noch winterlichen Frühling in die hochsommerliche Hitze hinein, wie ich es in Çanakkale erlebt habe. Ich kann versuchen, Poetisches über eigene ästhetische Erfahrungen zu vermitteln und mit der Aufgabe bzw. Anregung, solches in Worte zu fassen, literarisch erfahrbar zu machen.

\author{
Bunt sind schon die Wälder, \\ gelb die Stoppelfelder, \\ und der Herbst beginnt. \\ Rote Blätter fallen, \\ graue Nebel wallen, \\ kühler weht der Wind. [...]
}

Die Natur wird idealisiert und verklärt in der bürgerlichen Dichtung von Johann Gaudenz von Salis-Seewis (1762-1834), dessen ,Bunt sind schon die Wälder...' (1782), vertont (mit einer Melodie versehen), zu einem sehr populären Lied wurde (der Text ist hier stark gekürzt). Hier bekommt der ,Herbst ${ }^{\star}$ ein Gesicht, welches aus der Natur gewonnen ist. Es ist das traditionelle Bild, welches die deutsche Dichtung bis in die Gegenwart beschäftigt. Die Blätter der Bäume werden bunt - das assoziiert (verbindet sich mit den Gedanken von) Reichtum und Ernte. Aber diese Blätter fallen, Nebel und Kälte künden sich an. Damit assoziiert man in der Dichtung die Vergänglichkeit und den Tod. Der Herbst bekommt ein Gesicht, das auf den nahenden Winter ausgerichtet ist.

Ich stelle mir vor, dass die nächste Stufe (vielleicht einer Lehrveranstaltung im Abschlusssemester) die Vermittlung von Liebeslyrik wäre. Ein möglicher Ausgangspunkt ist

\footnotetext{
${ }^{9}$ Daraus entstand: Holzapfel, Otto (2006), „Herbstgedichte - Achtzehn Tage im Herbst: Ein poetisches Tagebuch als Weg zum Gedicht“. In: Holzapfel, Otto: Liedverzeichnis [Hildesheim: Olms, 2006] (erweiterte OnlineFassung, jeweils im Update, über die Homepage ,Volksmusikarchiv des Bezirks Oberbayern') । Textinterpretationen $\backslash$ Teil 5, S. 103-126.
} 
dabei die Schilderung, aus welchen Wurzeln sich der mittelalterliche deutsche Minnesang nährt, den wir ab etwa 1150 kennen. Dabei kann man beispielsweise über die nordfranzösische Trouvère-Lyrik auf die provenzalische Trobador-Lyrik des frühen 12 . Jahrhunderts zurückverweisen und vor allem (so eine der Theorien zur Entstehung des Minnesangs) auf arabische Vorbilder in Spanien, im maurischen Andalusien.

Könnte man nicht so etwas wie ,Stolz' vermitteln, dass wir es hier (wahrscheinlich) mit einer sehr frühen Form des ,Orientalismus ${ }^{610}$ (den man in diesem Fall allerdings nicht so nennt) zu tun haben, und zwar in einer vergleichbaren Situation, die auch das 18. Jahrhundert kennzeichnet? Obwohl das arabische Spanien nicht gerade mit freundschaftlichen Methoden ,zurückerobert' wurde (aus der Sicht der Franken: die ,Reconquista' begann zum Beispiel mit dem Königreich León 1037; der Fall von Granada war erst 1492), so konnte sich offenbar (bzw. vielleicht) doch über die kriegerischen Grenzen hinweg ein Kontakt aufbauen, der den ästhetischen Sinn für arabische, maurische Liebeslyrik in die neue höfische Gesellschaft in den eroberten Regionen hinüberrettete.

Als Beispiel wähle ich ein berühmtes Freundschaftsgedicht, das sich in einer um 1200 geschriebenen Handschrift in mittelhochdeutscher Sprache findet. Die Dichterin dieses Textes kennen wir nicht.

\author{
Dû bist mîn, ich bin dîn: \\ des solt dû gewis sîn; \\ dû bist beslozzen in minem herzen, \\ verlorn ist daz slüzzelîn: \\ dû muost och immer darinne sîn.
}

Du bist mein, ich dein... Wir sind gegenseitig eng verbunden; dessen sollst du gewiss sein, darauf kannst du dich verlassen. Du bist in meinem Herzen eingeschlossen. Das Schlüsselchen dazu ist verloren: Du musst auch immer darinnen bleiben. - Aus meinem ,Liedverzeichnis ${ }^{6}$ kann man weitere Daten dazu übernehmen: ${ }^{11}$ Man spricht von einer Liebesliedformel (ursprünglich ging es um Freundschaft), die zur traditionellen Redensart geworden ist. Im Anschluss an die Minnedichtung hat der Vers in der Kunstdichtung und in der Volksüberlieferung bis in die Gegenwart verbreitet und erhalten, im Volkslied zum Beispiel als Vierzeiler „Mein Herz und dein Herz sind zusammengebunden, der Schlüssel ist verloren, wird nimmer gefunden.“ In einer Handschrift von etwa 1747 steht „Du bist mein ich bin dein, mir soll auch nichts liebers sein." Und aus der Sammlung historischer Bildpostkarten, die an der Universität von Osnabrück angelegt wurde ${ }^{12}$, übernehme ich als Abbildung eine Postkarte von etwa 1917:

\footnotetext{
${ }^{10}$ Vergleiche dazu neuerdings Ercan, Cemile Akyildiz/Balci, Yasemin/Öztürk, Ali Osman (Hg.) (2019): Ex Oriente Lux: West-östlicher Kulturtransfer. Berlin: Logos Verlag (Germanistik in der Türkei, 1). ISBN 978-38325-4915-2 (mit weiteren Hinweisen).

${ }^{11}$ Holzapfel, Otto: Liedverzeichnis [Hildesheim: Olms, 2006] (erweiterte Online-Fassung 2020 über die Homepage ,Volksmusikarchiv des Bezirks Oberbayern') $\backslash$ Lieddatei.

12 Bildpostkartensammlung der Universität Osnabrück; bildpostkarten.uni-osnabrueck.de [aufgerufen im Dezember 2018]
} 


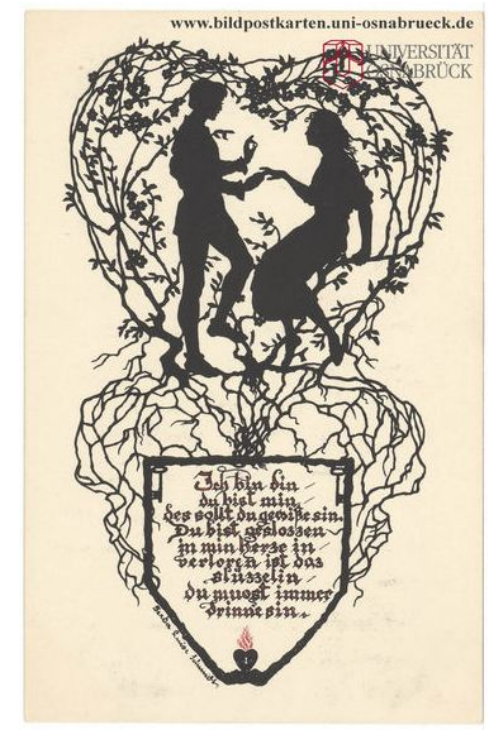

In einem Gutachten zu diesem Beitrag wird eine „Schlussfolgerung“ vermisst. Wie sollte diese aussehen? In der (nachgereichten) Einleitung (der Beitrag insgesamt in beiden Teilen wurde vor vielen Monaten geschrieben) steht einiges, was so verstanden werden könnte. Ich [Otto Holzapfel] habe darin angedeutet, dass wir uns vielleicht auf neue Lehr- und Lernstrategien einstellen müssen, falls Lehre und Prüfung fast ausschließlich online vorgehen muss. Das würde meines Erachtens z. B. auf beiden Seiten eine neue und vertrauenswürdige ,Ehrlichkeit' bedeuten, in der sich Lehrende und Studierende weniger hierarchisch bedingt gegenübertreten, sondern gemeinsam Sprache und Literatur, entdecken'. Der Lehrende wird eher ,Wege" aufzuzeigen haben, als Vorschriften zu machen, der Lernende wird eher versuchen müssen, sich mit Entdeckerfreude Kenntnisse in Sprache und Literatur anzueignen, weniger auf ,gute Noten' zu schielen. Ob das schließlich möglich sein wird und erfolgreich bleibt, kann man bezweifeln. Aber ein Weg, sich in unangestrengter Weise Literatur und Sprache zu nähern, ist ,Poetologie‘ allemal.

\section{Literaturverzeichnis}

Bildpostkartensammlung der Universität Osnabrück; bildpostkarten.uni-osnabrueck.de [aufgerufen im Dezember 2018]

Ercan, Cemile Akyildiz/Balci, Yasemin/Öztürk, Ali Osman (Hg.) (2019): Ex Oriente Lux: West-östlicher Kulturtransfer. Berlin: Logos Verlag (Germanistik in der Türkei, 1).

Gencer Çıtak, Özlem (2019): Zur Entwicklung von Gesprächskompetenzen im Deutschen als Fremdsprache. Eine longitudinale Studie anhand der Germanistikstudenten in der Türkei. Berlin: Logos Verlag (Germanistik in der Türkei, 5).

Holzapfel, Otto (2006), Herbstgedichte - Achtzehn Tage im Herbst: Ein poetisches Tagebuch als Weg zum Gedicht. In: Holzapfel, Otto: Liedverzeichnis [Hildesheim: Olms, 2006] (erweiterte Online-Fassung, jeweils im Update, über die Homepage, Volksmusikarchiv des Bezirks Oberbayern') Textinterpretationen $\backslash$ Teil 5. 103-126.

Holzapfel, Otto (2016): Erfahrungen mit Wikipedia.de. In: [türkische Internet-Zeitschrift] Diyalog. Interkulturelle Zeitschrift für Germanistik 2016/1. 99-118. 
Holzapfel, Otto (2018): Vom Vergnügen ein älteres Fach zu lesen. Eine Diskussion im Grenzgebiet von Naturund Geisteswissenschaft. In: [türkische Internet-Zeitschrift] Anadolu University Journal of Education Faculty (AUJEF), Special Issue August 2018 = Festschrift Seyyare Duman. 179-184.

Staiger, Emil (1982): Die Kunst der Interpretation. Studien zur deutschen Literaturgeschichte. Zürich: Atlantis 1955; 5., unveränderte Auflage München: Deutscher Taschenbuch-Verlag. 